\title{
An Intelligent Knowledge Representation of Smart Home Energy
} Parameters

\author{
Mario J. Kofler ${ }^{*}$, Christian Reinisch, Wolfgang Kastner ${ }^{1}$ \\ Vienna University of Technology, Automation Systems Group, Vienna, Austria \\ * Corresponding author. E-mail: mjk@auto.tuwien.ac.at
}

\begin{abstract}
Homes in today's world tend to include more and more electrically powered devices. Much effort is put on improving these facilities, but their integration towards a smart home often remains unconsidered. While there are some promising approaches to integrate devices with the help of knowledge bases, they are still not fully convincing. In all cases they fail to cover the energy behavior of installed devices which is a severe shortcoming with respect to the increasing energy demand of a home. As most residents are still unaware where the energy is consumed and which actions eventually lead to a lower demand, an energy related knowledge representation is of importance. This paper proposes such an energy knowledge base modeled as ontology. This artifact comprises a comprehensive collection of miscellaneous energy related information and allows home automation systems to make intelligent decisions upon this knowledge. Using the ontology, energy consumption in the home itself can now be optimized by executing intelligent control strategies that incorporate and exploit the additional knowledge in their algorithms. Likewise, also renewable energy suppliers are represented and may be considered by a smart home system in order to reduce the overall ecological footprint of the residents and provide additional services for home control.
\end{abstract}

Keywords: Energy Parameters, Smart Homes, Ontologies

\section{Introduction}

The deployment of automation technology in the home offers several attractive benefits, among them most prominently increased energy (or even resource) efficiency, improved residential comfort and peace of mind for the home owner. As private households are undoubtedly one of the main energy consumers today, also a positive effect for the environment can be expected if energy consumption is reduced. In the last decade, smart homes have emerged as the keyword for such automated dwellings. The vision is a house populated by a multitude of devices (actuators and sensors) that cooperate in an intelligent way to control different domains of the home such as lighting/shading, heating/ventilation/airconditioning but also home appliances and consumer electronics. While in building automation well established solutions have already existed for a longer time, additional challenges arise for systems that need to be tailored to the needs of private households: In this domain, integration of diverse appliances into a homogeneous system is far from trivial due to different interfaces, usage paradigms and operation modes. Additionally, the intelligence promised by smart homes requires tailored use cases and scenarios to be developed and offered by the future systems. Consider, for example, a smart home system automatically scheduling a dishwasher to start when energy from renewable energy sources becomes available, e.g., when the sun is shining on a photovoltaic installation or once some energy provider offers cheap energy. Such a system could also be the central point to integrate demand side management [1] applications into the house, e.g., by shifting energy intensive operations to a more convenient point in time. These use cases require not only all devices to be interoperable but also demand some understanding of the current state of the affected environment. Information about the building, its embedded devices, its tenants and their

\footnotetext{
${ }^{1}$ This work was funded by the HdZ+ fund of the Austrian Research Promotion Agency FFG under the project 822170.
} 
behavior as well as of the inside and outside conditions must be available and represented in some way for the smooth and successful operation of the smart home system.

\section{Motivation}

More and more energy facilities in modern buildings become interlinked in order to allow advanced control over various parts of the house. Up to now, however, only basic services are featured: lights and shutters can be linked via pre-configured scenes, or, for example, a central "OFF" function can be provided. More intelligent functions like ensuring comfort in a residential home while at the same time behaving energy efficiently are not feasible yet. The reasons for this are manifold, but one of the most important issues is the interoperability of devices. This integration is often not guaranteed due to the heterogeneity of underlying technologies. An orchestration of their services can often just be achieved through interconnection using gateways which are difficult to configure and may still limit interdevice services [2]. Once integrated, all devices may be controlled and operated through a central home control system. However, this does not automatically imply that also all data of the devices becomes available throughout the integrated system. Based on these current shortcomings of smart homes, two main challenges can be identified: the need for an integrated system where all devices can equally participate, and some storage facility that provides pervasive access to all kinds of data originating from devices, the smart home or other sources. Thus, an abstract view on the underlying technologies is desirable to facilitate the integration of different building automation devices and also home applications. Further, often it is not known to a resident how much energy certain devices in the household consume. Many devices also waste energy when they are currently not in use e.g., during their stand-by times. To reduce these idle times it would help a smart home to know about the occupancy of rooms and during which times facilities in the home are mainly used. Especially in the case of consumer electronics like TVs it makes sense to unlink them from the power grid during times when the residential home is not occupied and just turn them back into stand-by mode when usage is expected. For household appliances like dishwashers or washing machines it would be beneficial to know how to schedule tasks with respect to the energy supply side. This way, peak loads on the power grid can be reduced and at the same time the environmentally friendliest energy provider can be chosen. The definition of energy tariffs and providers in the knowledge base of the smart home therefore allows yet unconsidered improvements with respect to energy consumption. The representation of such facts needs to be sophisticated and open to changes, because it is not only likely that new and probably unknown devices are added to the smart home, but also information about energy providers and their tariff schemes are changing frequently. These difficulties are addressed by the realization of a knowledge base for smart homes that is proposed in the following chapter.

\section{Methodology: An Ontology for Smart Homes}

The intelligent information representation in smart homes is necessary, not least because of the vast amount of influences to be considered for an energy efficient operation of the building. To model the data dependencies in an expressive way, the representation as OWL ontology is proposed. OWL is a recommendation of the World Wide Web Consortium (W3C) [3] and its Semantic Web Initiative. The Web Ontology Language bases its form and representation on a formal logic called Description Logic (DL) [4]. With this formal grounding, relationships and concepts existent in DL as well as the possible logical implications can be used in OWL for modeling the represented domain in a sophisticated manner. This way, more complex structures can be expressed than in alternative possibilities like relational database systems. Opposed to classical database schemes, the well-defined 
logics of DL further allow reasoning over explicitly modeled facts in the knowledge base. This way, the inference of new information out of existing data becomes possible, and queries can be already stated in the knowledge base itself (cf. Sect. 4.2). Also the consistency of the knowledge representation can be assured automatically by the reasoning mechanism, when considering for example the addition of new concepts and relationships. In the case of smart homes, all knowledge can therefore be well organized and brought into an intelligent structure that subsequently can be accessed by the smart home system.

\subsection{Ontology Overview}

The proposed knowledge base consists of several modules which contain different kinds of parameters important for an energy efficient operation of smart homes (Fig.1).

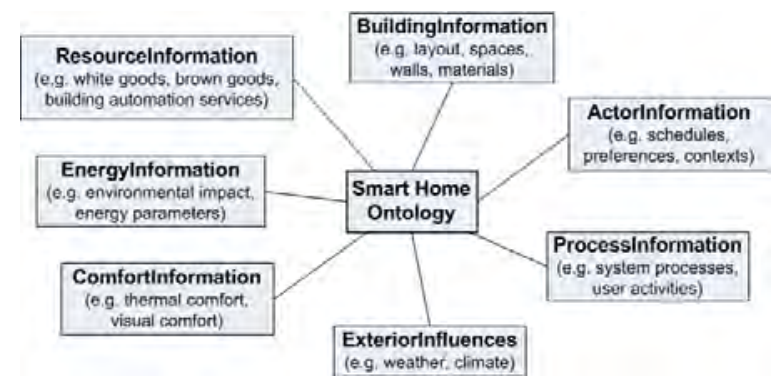

Fig. 1. Smart Home Ontology Main Modules

These different parts are, for example, a building representation including information about architecture and building physics, a user part with preferences as well as an exterior influences module holding for example weather data. In this work, focus is put on the resources and energy parts of the knowledge base: The resource representation describes the available facilities and their characteristics. The entire home environment and equipment has to be modeled in the knowledge base for a control system to have a complete view of the operable world, i.e., the building and its devices. The energy representation is an important source of information about energy demand and energy supply of the smart home. One of its purposes is to allow a software system to base its operational decisions on the status of the connected facilities in the smart home. Further, a representation of energy providers and energy tariffs enables an ecological and economical use of different energy forms such as electricity or district heating, with respect to renewability and energy costs. The next section describes these two parts in detail and explains the benefits of expressing facility and energy parameters as a linked knowledge store.

\subsection{Facilities and Home Automation Systems}

In home and building automation (HBA), the interaction of numerous kinds of devices is desirable. In most cases integration is not directly possible because of the heterogeneity of different home automation network standards. With DomoML [5] and DogOnt [6], there already exist two approaches that propose the use of ontologies in this context. DomoML is one of the first proposals structurally modeling household appliances with the help of ontologies. While DogOnt reuses certain ideas of this taxonomy, it tries to overcome limitations of DomoML. As ontology reuse [7] is highly recommended in ontology design, the DogOnt ontology was chosen as a starting point for the resource module of the proposed knowledge base. While not perfectly suitable for reuse, the DogOnt implementation provides an extensive and sophisticated representation of building facilities, functions and possible modes of operation. The authors of DogOnt put the focus of their knowledge base on the 
intelligent integration of home facilities and automation components and aim at building automation service interoperability [6]. However, energy related issues like energy supply or demand of mapped home automation systems and home appliances have not been considered. Also, building information has only been rudimentarily treated. These facts make the DogOnt ontology a good candidate for integration into the proposed smart home ontology. Referring to Fig.1, the DogOnt ontology can represent the resource part, while interfacing with the more detailed building information branch as described in [8] and the newly developed energy representation module presented in Chapter 4. However, the ontology of the DogOnt project contains several severe design flaws, especially with respect to ontology normalization. Among others, important ontology normalization steps like avoiding asserted polyhierarchies and instead using hierarchical tree structures have been ignored by the creators of DogOnt. Nevertheless, as stated in [9], a normalized ontological representation significantly raises the reusability and is therefore considered as key requirement for large ontologies. As a consequence, the DogOnt representation is first adapted into a semi-normalized form by reformulation of comparatively weak parts of the ontology while making a tradeoff between fully normalized form and practicability. The key design focus of this reformulation is to keep the original hierarchy as far as possible, but, for example, to only allow polyhierarchies to be automatically asserted by a DL reasoner (e.g., Pellet [10]). This normalized version of DogOnt is subsequently integrated into the proposed knowledge base.

\section{Results: Energy Information Representation}

In order to describe information from the energy domain for a smart home system, some important concepts need to be modeled. These so-called top-level concepts contain the following necessary classifications:

- Energy providers: This concept comprises all external energy suppliers providing some form of energy for the residential home.

- Energy tariffs: The tariffs that are charged by an energy provider to supply a certain energy type.

- Energy types: The different energy types that are available and are either supplied by energy providers, or used as source of energy to produce some secondary energy.

- Energy facilities: All energy consuming or energy producing applications that are installed in a smart home.

- Energy properties: This concept contains information needed to model energy demand and supply as well as energy costs.

Energy representation, as module of the proposed smart home knowledge base (cf. Fig.1), therefore keeps a wide variety of different parameters useful for the energy efficient operation of a home. To provide the system with a general notion of energy, a classification of energy types is needed. This classification has to be tailored to the needs of a smart home with respect to modeled energy types. Therefore, a distinction between final energy, energy sources and useful energy was taken (Fig. 2). This distinction reflects the varied usage of energy types viewed from the providing side as well as from the consuming side.

The concept EnergySource is used to classify different energy providers as explained in Section 4.1 and follows the general definition of sources of energy. Two distinctions are made: The first distinction is into primary and secondary sources of energy. Electricity, for example, is a secondary energy source because it has to be gained through some primary energy source. The second distinction into renewable and non-renewable sources is especially 
important for resource-efficient energy consumption in a smart home. These two concepts are finally super-concepts of the actual energy sources. Some design decisions are made in order to classify sources of energy: For example, nuclear power is assigned to the non-renewable branch as concept Nuclear, because also nuclear waste is taken into account as some type of environmental pollution.

The other two main concepts are FinalEnergy, and UsefulEnergy which both represent energy that is available for consumption in the smart home. A distinction between these two concepts is realized such that final energy contains energy types that can still be transformed to other energy forms inside the smart home while useful energy types are inconvertible. For example, gas as final energy can be used directly by a gas oven or, with the help of a gas heater, can be transformed to heat, which can subsequently be seen as useful energy.

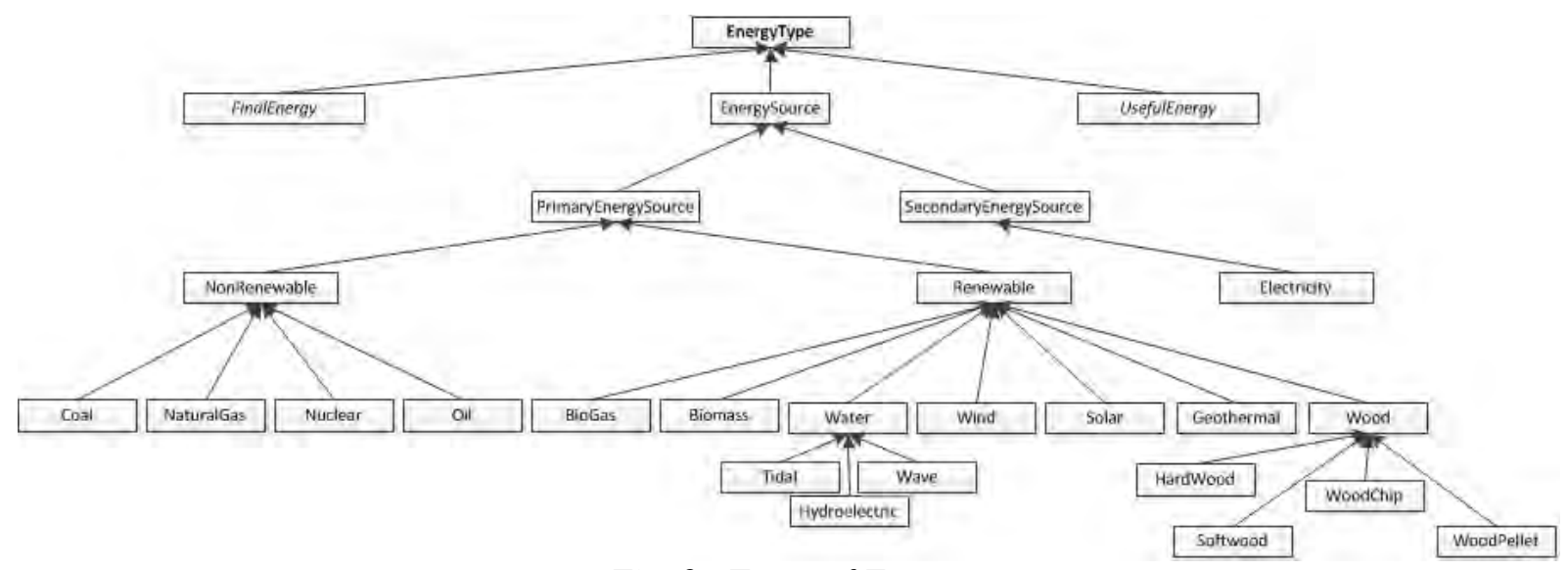

Fig. 2. Types of Energy

These two classifications of energy types do not have subclasses like the energy source branch, but merely contain concrete values which correspond to different forms of energy. This modeling technique is chosen, because for the appliance it does not make a difference if a green or non-green provider supplies the energy, however for the ecological footprint of the smart home user it does (cf. Sect. 4.2). The concrete members of the concept FinalEnergy are for example Coal, ElectricEnergy, Gas and Wood, while the concept UsefulEnergy has the members Heat, Cold, Light and Water. These groups can always be enlarged or narrowed, according to which end energies should be covered by the smart home system. As can be seen, some energy types occur twice in the knowledge base: once as energy sources and secondly as members of one of these two concepts (cf. Fig.2). The reason for this is that basically two viewpoints are being represented in the energy ontology: The demand side and the supply side. These two sides need to have a different idea of energy, because there exist energy sources that can be used by an energy provider to generate secondary energy but can also be directly used for consumption in the smart home as final energy. Also for the UsefulEnergy concept such a special case can be found: the specific resource Water on one hand acts as a source of energy to generate hydroelectricity, on the other hand it can be directly seen as useful energy in the smart home with different water providers and tariffs. Therefore, the classification shown in Figure 2 is considered as the one with least redundancies and most practical use. The benefits of the realization of final energy and useful energy types as individual values instead of concepts are further discussed in Section 4.2.

On one side it is important to model the demand and supply facilities which are available in the smart home itself. Certainly, it is a better choice to use energy produced by home facilities from solar radiation and geothermal heat than having to rely on the supply of energy from 
energy providers. On the other side it is also necessary to keep knowledge about different energy providers and their conditions in case energy demand exceeds homemade supply. Therefore, energy information representation is divided in two main axes which contain facilities in the smart home itself, i.e., the demand side and energy producing facilities, and the supply side like energy providers and tariffs, respectively. The following two sections go into detail and describe the certain constructs that have to be modeled for these two main parts. Although a description of the whole energy knowledge representation would go beyond the scope of this paper, important constructs and design paradigms are discussed in order to demonstrate the representation of energy demand and supply in the proposed knowledge base.

\subsection{Energy Providers and Supply Side}

With the ongoing liberalization of energy markets, knowledge about different energy providers and their tariffs are a valuable addition to the smart home ontology. It is assumed that like in the electric energy market also other markets will be liberalized in the future and therefore a variety of energy suppliers is classified in the proposed knowledge base (Fig.3).

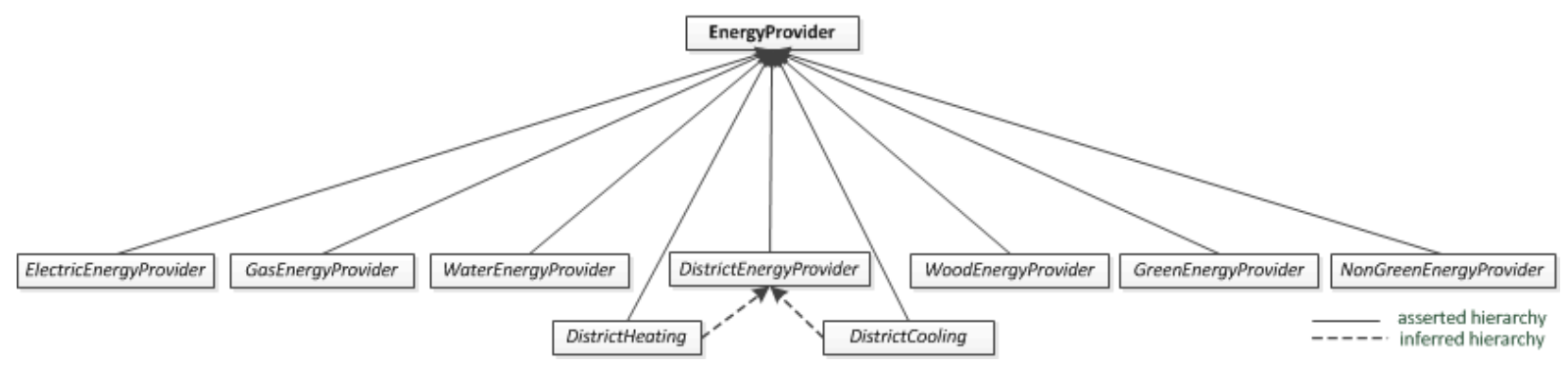

Fig. 3. Classification of Energy Providers

This conceptualization of energy providers comprises different energy forms as well as the distinction between green and non-green suppliers. Reasoning on this hierarchy with a DLreasoner, makes inferred hierarchies possible as shown in Figure 3 for district energy providers. Furthermore, reasoning can classify newly added individual energy providers and associate them with the respective subclass. This quality of an ontological representation can aid the characterization of green and non-green energy providers with respect to the way they supply energy. For example, some electricity provider which provides electric energy only through hydropower will become a member of the classes ElectricEnergyProvider and GreenEnergyProvider. In case this energy provider adds some non-green way to provide energy (e.g., nuclear power), the classification will be automatically corrected by the reasoning mechanism and the company will further be listed as NonGreenEnergyProvider. In addition to the way how suppliers generate energy, it is needful to know the different kinds of energy tariffs. Of course not every company has the same rates for energy supply and also different tariff switching times can exist which have to be considered in the knowledge base too. Therefore, a general notion of time is required which is achieved by integrating the OWLTime ontology for time representation [11]. With the reuse of this time ontology, characterization of tariffs according to their active times becomes possible. Together, energy provider and energy tariff form the main concepts of the supply side. They can further be used by a smart home control system to choose the environmentally friendliest and monetarily optimal energy supply at a specific point in time.

\subsection{Energy Facilities and Demand Side}

Energy facilities with respect to the smart home are all appliances which either consume or produce energy. For the facilities represented in the ontology, the actual energy consumption 
as well as the maximum energy consumption per defined state of operation is stored. Further, it is important for an autonomous system to know if a certain facility needs permanent power supply. With this information, an intelligent system can for example unlink appliances from the power grid when they are not immediately needed.

The connection between energy demand side and energy supply side is made via an ontology design pattern called class-individual mirror described in [12]. For this design pattern, the final energy types already explained at the beginning of this chapter act as pivotal elements. The example in Fig. 4 shows the application of the pattern for these two parts of the ontology.
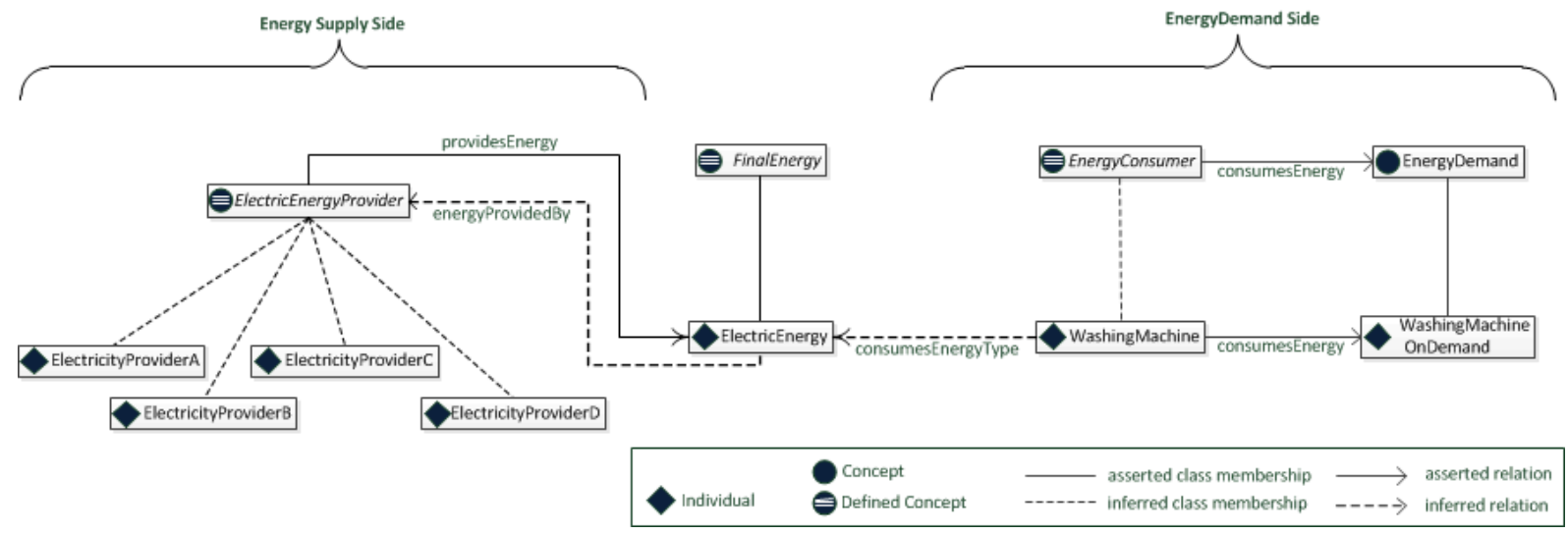

Fig. 4. Connection between Energy Providers and Energy Facilities

The benefit of using this pattern is that the pivotal ElectricEnergy element holds information from the energy supply side. This can be used for choosing the electricity provider on an appliance level: If the depicted washing machine is scheduled to start at a certain point in time, the home automation system just needs to know which energy type it consumes. The ElectricEnergy element already holds information about which energy providers supply the energy by the energyProvidedBy relation that has been inferred by the DL-reasoner. Energy tariffs and other properties of the energy providers like if they are green or non-green suppliers can subsequently be retrieved from the ontology by the properties that have been defined for each electricity company. This way, important queries have already been modeled in the knowledge base, which represents a clear advantage over classical database systems. Among other things, this leads to a higher independency between the data representation and the software system.

\section{Conclusion and Outlook}

This paper proposed a central knowledge base which is mandatory to enforce novel energy efficiency control strategies in smart homes. It was shown that ontologies are a well suited technology to use as smart home knowledge representation. The ontology constructs as well as the formal grounding in Description Logics allow the depiction of more detailed and interconnected information than known from classical information representation systems. Additionally, because of the foundation in logics, reasoning on stored facts becomes possible which allows inference of new information and guarantees the knowledge model's consistency. As a proof of concept, the part of energy related data was modeled as OWL ontology. Special focus was given to the domains demand side and supply side, where in particular the interrelations between the parts were discussed extensively. 
A practical example of the application of the energy knowledge base is the energy efficient operation of household appliances and consumer electronics. Information about scheduled programs and desired finishing times can, for example, be used by a software system to derive which renewable energy provider offers the optimal tariff for the planned task. Furthermore, time slots for the execution of tasks give the system the ability to wait until off-peak electricity is offered, thus on one hand saving money for the customer, while on the other hand behaving environmentally friendly by consuming excess energy.

Next steps regarding the presented resource and energy ontologies will concern their integration into a software framework as well as the definition of an interface that allows autonomous smart home control systems to easily access the knowledge store. Finally, other important parts of the comprehensive knowledge base for smart homes will be defined, modeled and constantly refined.

\section{References}

[1] P. Jazayeri, A. Schellenberg, W.D. Rosenhart, J. Doudna, S. Widergren D. Lawrence, J. Mickey, S. Jones, A Survey of Load Control Programs for Price and System Stability, IEEE Transactions on Power Systems, Vol. 20, No. 3, 2005, pp $1504-1509$.

[2] W. Kastner, G. Neugschwandtner, S. Soucek, H. M. Newman, Communication Systems for Building Automation and Control, Proc. of the IEEE 93(6), 2005, pp 1178 - 1203.

[3] W3C Recommendation, OWL 2 Web Ontology Language Document Overview, http://www.w3.org/TR/owl2-overview/, 2009, (online).

[4] F. Baader, D. Calvanese, D. L. McGuinness, D. Nardi, and P. F. Patel-Schneider (Eds.), The Description Logic Handbook: Theory, Implementation, and Applications, Cambridge University Press, 2003.

[5] L. Sommaruga, A. Perri, F. Furfari, DomoML-env: An ontology for Human Home Interaction, Proc. of the 2nd Italian Semantic Web Workshop, 2005.

[6] D. Bonino, F. Corno, DogOnt - Ontology Modeling for Intelligent Domotic Environments, Proc. of the 7th International Conference on The Semantic Web, 2008.

[7] E. Paslaru Bontas, M. Mochol, R. Tolksdorf, Case Studies on Ontology Reuse, Proc. of the 5th International Conference on Knowledge Management , 2005.

[8] M. J. Kofler, W. Kastner, A Knowledge Base for Energy-Efficient Smart Homes, Proc. of the International Energy Conference ENERGYCON, 2010.

[9] A. L. Rector, Modularisation of Domain Ontologies Implemented in Description Logics and related formalisms including OWL. Proc. of the International Conference on Knowledge Capture, 2003, pp. 121-128.

[10]E. Sirin, B. Parsia, B. Cuenca Grau, A. Kalyanpur, Y. Katz, Pellet: A practical OWL-DL reasoner, Journal of Web Semantics, Volume 5, Issue 2, 2007, pp 51 - 53.

[11]F. Pan, A Temporal Aggregates Ontology in OWL for the Semantic Web, Proc. of the AAAI Fall Symposium on Agents and the Semantic Web, 2005.

[12]D. Allemang, J. Hendler, Semantic Web for the Working Ontologist, Morgan Kaufmann Publishing/Elsevier, 2008, pp. 251 - 253. 